\title{
Hans Maier
}

\author{
Compelle intrare \\ Rechtfertigungsgründe für die Anwendung von \\ Gewalt zum Schutz und zur Ausbreitung des Glaubens in der \\ Theologie des abendländischen Christentums
}

\section{Die Ausgangslage}

Der sittlichen Pflicht, die Wahrheit zu suchen und der erkannten Wahrheit zu folgen, kann der Mensch nur in völliger Freiheit entsprechen. Daher verbietet sich staatlicher oder kirchlicher Zwang in Religionsdingen von selbst. Diese Einsicht war in der christlichen Theologie schon früh verbreitet und ist in vielen patristischen Texten belegt. In ihnen spiegelt sich die Befreiung des christlichen Glaubens vom Druck der antiken Staatskulte: An die Stelle kollektiver Rituale trat immer stärker das persönliche Bekenntnis von Einzelnen.

In Sachen Religion allein der eigenen Überzeugung gemäß zu handeln, ist deshalb nach Tertullian ein Menschenrecht. Religion leidet keinen Zwang, am wenigsten durch Religion selbst - nec religionis est cogere religionem ${ }^{1}$. Denn daß jemand unter Zwang etwas verehrt, das er im Ernst gar nicht verehren will, sagt Lactanz, kann nur zu Heuchelei und Simulantentum führen ${ }^{2}$. Im Kirchenrecht entwickeln sich in den mittelalterlichen Jahrhunderten entsprechende Rechtsgrundsätze. So hält Gratian daran fest, daß „zum Glauben niemand zu zwingen ist“ (ad fidem nullus est cogendus) $)^{3}$, und Thomas bekräftigt diesen Satz mit dem Hinweis, der Glaube sei Sache des (freien) Willens ${ }^{4}$. Entsprechende Festlegungen der Kanonistik reichen bis in die Neuzeit, ja bis in die Gegenwart hinein. Sowohl das alte wie das neue Kirchenrecht halten bis heute strikt an der Ablehnung des Glaubenszwanges fest. Der Codex Iuris Canonici von 1983 enthält den Satz: „Niemand hat das Recht, Menschen zur Annahme des katholischen Glaubens gegen ihr Gewissen durch Zwang zu bewegen. “5

1 Tertullian, Ad Scapulam (ed. Bas. 1591) 498.

${ }^{2}$ Lactantius, Epitome, c. 54.

${ }_{4}$ Decretum Gratiani, pars II, c. 23, q. 5, c. 33.

4 Thomas von Aquin, Summa theologica II-II, 10, 8.

${ }^{5}$ Homines ad amplectendam fidem catholicam contra ipsorum conscientiam per coactionem adducere nemini umquam fas est. CIC can. $748 \$ 2$. Hierzu Winfried Aymans, Kanonisches Recht, Lehrbuch aufgrund des Codex Iuris Canonici (Aymans-Mörsdorf III, Paderborn 2007) 8f. 
Gleichwohl ist nicht zu übersehen, daß es in der Geschichte des Christentums immer wieder Gewalt in Sachen Religion gegeben hat, Gewalt auch „im Namen der Religion" - das springt jedem Betrachter, der die Zeugnisse ansieht, ins Auge. Über Macht und Zwang in der Kirche, über die Gewalttaten von Christen, über die sogenannte „Kriminalgeschichte des Christentums“6 gibt es mittlerweile eine umfangreiche, oft recht polemische Literatur. Die Geschichte des Christentums wird jedoch heute nicht nur von außen, von Gegnern und Feinden, kritisch in den Blick genommen. Auch unter Christen spielt die Auseinandersetzung mit der eigenen Vergangenheit eine wichtige Rolle. Insbesondere die katholische Kirche hat in jüngster Zeit vielfältige Initiativen ergriffen, fragwürdige Vorgänge ihrer Geschichte genauer zu erforschen - nicht in apologetischer Absicht wie meist in früheren Zeiten, sondern durchaus mit dem Willen zu Aufklärung und Remedur. So hat Papst Johannes Paul II. in seiner berühmten Vergebungsbitte am 12. März 2000 das voraufgehende allgemeine und besondere Schuldbekenntnis auf die gesamte Vergangenheit der Kirche bezogen. Zweitausend Jahre kirchlicher Praxis bei der Verkündung des Evangeliums stehen zur Debatte. Das Fazit in den Worten des Papstes: „... oft haben die Christen das Evangelium verleugnet und der Logik der Gewalt nachgegeben... Vergib uns!“7

Worin gründet solche Gewalt im Christentum, die doch offenkundig im Widerspruch steht zu der früh bekräftigten Gewaltfreiheit in Sachen des Glaubens? Drei Beweggründe dürfen genannt werden. Da ist erstens der Schutz der Glaubensgemeinschaft gegen Unglauben und Irrglauben, der in seiner konkreten Ausgestaltung, besonders beim Vorgehen gegen Häretiker und Ketzer, nicht selten zu Intoleranz und Gewalt geführt hat. Ein solcher Schutz ist notwendig in jeder geistigen Gemeinschaft - aber wo liegen seine Grenzen? Da ist zweitens die Verteidigung des Glaubens gegen - wirkliche oder vermeintliche - Angriffe von seiten der Ungläubigen, eine Verteidigung, die immer wieder dazu führte, daß man über die bloße Abwehr hinausging und am Ende in Gewalt verfiel, in eine nicht endende Spirale von Ausschreitungen, Pogromen, Kämpfen, Kriegen. Das historische Schulbeispiel sind die Kreuzzüge; aber auch das Verhältnis zwischen Christen und Juden im Abendland ist auf weite Strecken der Geschichte von dieser Dialektik geprägt. Und da ist drittens die Ausbreitung des Glaubens in der Welt, mit der das Christentum den biblischen Missionsbefehl („Geht hinaus in alle Welt...“) zu erfüllen versuchte. Im Zug der missionarischen - und später kolonialen - Erschließung der Welt durch das christlich geprägte Europa wurde die religiöse Selbstbestimmung unterworfener Völker, die Freiwilligkeit ihrer Zustimmung zum christlichen Glauben oft massiv verletzt - bis zu dem Punkt, daß die Weigerung, den

${ }^{6}$ So das gleichnamige dreibändige Werk von Karlheinz Deschner (Reinbek 1986-1990); zur Kritik vgl. Hans Reinhard Seeliger, Kriminalisierung des Christentums? Karlheinz Deschners Kirchengeschichte auf dem Prüfstand (Freiburg 21993).

7 Pressemitteilungen der Deutschen Bischofskonferenz: Vergebungsbitte von Papst Johannes Paul II. Allgemeines Gebet, Schuldbekenntnis und Vergebungsbitte beim Pontifikalgottesdienst am 12.03.2000 in St. Peter in Rom (das Zitat 5). 
christlichen Glauben anzunehmen, als Unrecht gegen Gott erschien und Kriege gegen die Eingeborenen zu Glaubenskriegen wurden ${ }^{8}$.

An all diesen Vorgängen ist freilich nicht nur die Kirche, sondern auch der Staat beteiligt. Das muß man bei der Analyse der Vorgänge stets bedenken, will man nicht in monokausale Zuschreibungen, in ungeprüfte Vor-Urteile verfallen. Zwar hat die christliche Theologie von Anfang an im Sinn des biblischen Zinsgroschengleichnisses argumentiert, sie hat stets unterschieden zwischen dem, was des Kaisers, und dem, was Gottes ist - eine Unterscheidung, die in den „theopolitischen“ Religionen der Antike naturgemäß keine Rolle spielte ${ }^{9}$. Doch in der Praxis hat man zwischen kirchlichen und weltlichen Zuständigkeiten oft weniger differenziert, als dies dem Stand der Theorie und Theologie entsprach ${ }^{10}$. Im historischen Ablauf ist es im Abendland immer wieder zu Rückbewegungen ins Gewohnte, zu unerwarteten Verdichtungen der alten Staat-Kirche-Einheit gekommen - zu Fortschreibungen des altrömischen Reichsmodells im Mittelalter oder zum „InstitutionenPaktieren“ zwischen Staat und Kirche in der Neuzeit. Manchmal wurde daraus ein regelrechtes Schwarzer-Peter-Spiel: Der Staat versteckte sich hinter der Kirche oder die Kirche hinter dem Staat.

Erst spät, nach den modernen Revolutionen, nimmt die Kirche Abschied vom Modell des „christlichen Staates“ und sucht und findet ihre eigene Freiheit. Und auch der Staat hört erst relativ spät auf, die Kirche nach seinem Bild gestalten und modellieren zu wollen (der letzte Versuch waren die Kulturkämpfe des 19. Jahrhunderts). Erst auf dieser Basis neuer (und nunmehr endgültiger!) Unterscheidungen setzt sich dann das Ideal wechselseitiger Unabhängigkeit von Staat und Kirche durch. Und erst damit wird dann auch die volle, nicht mehr durch Vorbehalte eingeschränkte Anerkennung der Religionsfreiheit möglich ${ }^{11}$.

\section{Das Corpus delicti: Compelle intrare}

Unter den theologischen Begründungen für Gewalt in Religionssachen hat die Figur des biblischen compelle intrare zurecht die größte Berühmtheit erlangt. Es ist

8 Joseph Höffner, Kolonialismus und Evangelium (Trier $\left.{ }^{2} 1969\right)$ 42ff., $62 \mathrm{ff}$.

9 Arnold A. T. Ehrhardt, Politische Metaphysik von Solon bis Augustin I-III (Tübingen 1959-1969); Hugo Rabner, Kirche und Staat im frühen Christentum (München 1961).

10 Grundsätzlich zum Gegenüber von „Konflikt" und „Loyalität" in der Geschichte des frühen Christentums: Paul Mikat, Konflikt und Loyalität. Bedingungen für die Begegnung von früher Kirche und römischem Imperium (Paderborn 2007). Zur Vorgeschichte der Toleranz: Klaus Schreiner, Gerhard Besier, Art. Toleranz, in: Geschichtliche Grundbegriffe 6 (Stuttgart 1990) 445-605; Rainer Forst, Toleranz im Konflikt. Geschichte, Gehalt und Gegenwart eines umstrittenen Begriffs (Frankfurt a. M. 2003).

11 John Courtney Murray, We hold these Truths. Catholic Reflections on the American Proposition (London 1960); Jérôme Hamer, Yves Congar (Hrsg), Die Konzilserklärung über die Religionsfreiheit, lateinischer und deutscher Text, mit Kommentaren von Pietro Pavan, Jan Willebrands, Émil-Joseph de Smedt u.a. (Paderborn 1967); künftig zitiert: Hamer, Congar, Konzilserklärung. 
eine überaus griffige Figur; Belege finden sich in der ganzen Kirchengeschichte: von Augustin bis zu den Reformatoren, von den spanischen Scholastikern bis zu Theologen der modernen Volksmission. Das compelle intrare bot über lange Zeiten einen unauffälligen, einen - wie es schien - legitimen und unanstößigen Einstieg in die schwierige Problematik Religion - Mission - Gewalt ${ }^{12}$.

Zunächst sei der einschlägige Text zitiert. Er steht im Lukasevangelium im 14. Kapitel, Vers 16-24. Dort ist die Rede von dem Hausvater, der ein großes Abendmahl bereitet und viele Gäste dazu einlädt. Doch die Geladenen fangen an, sich zu entschuldigen: Der eine hat einen Hof erworben (im Griechischen heißt es: einen Acker) und muß hingehen, um ihn anzusehen; der andere hat fünf Joch Ochsen gekauft und muß sie beschauen; der dritte hat eine Frau genommen und kann deshalb nicht kommen. „Und der Knecht kam zurück und berichtete das seinem Herrn. Da ward der Hausvater zornig und sprach zu seinem Knechte: Geh schnell hinaus auf die Straßen und Gassen der Stadt, und führe die Armen, Schwachen, Blinden und Lahmen hier herein. Und der Knecht sprach: Herr, es ist geschehen, wie Du befohlen hast; aber es ist noch Platz übrig. Und der Herr sprach zu dem Knechte: Geh hinaus auf die Landstraßen und an die Zäune und nötige sie hereinzukommen, damit mein Haus voll werde. Ich sage euch aber, daß keiner von den Männern, die geladen waren, mein Abendmahl verkosten soll."13

Die Stelle heißt im Vulgata-Text: ... et compelle intrare, ut impleatur domus mea. Griechisch lautet sie: kai anankeson eiselthein, hina gemisthe mou ho oikos. „Nötige sie hereinzukommen", das ist wohl die angemessene, sachlich am besten treffende deutsche Übersetzung, „Zwinge sie“ wäre zu stark, „bitte sie“ oder „fordere sie auf" wären zu schwach. Sowohl Luther ${ }^{14}$ wie Alliolii5 und die meisten katholischen Übersetzungen wie auch die Einheitsübersetzung von $1972^{16}$ verwenden den Ausdruck „nötigen“: Er trifft das lt. compellere (treiben, zusammentreiben) gut und noch besser das gr. anankazo, dessen Bedeutungsspektrum von „zwingen“ bis $\mathrm{zu}$ „überzeugen“ reicht.

Bei den griechischen und lateinischen Vätern hat dieses Gleichnis lange Zeit kaum eine Rolle gespielt. Seine theologische Nutzung und Indienstnahme setzt erst mit Augustinus ein - dann freilich mit großer Wucht und intensiver Langzeitwirkung. Um auch hier das Original zu zitieren: Der späte Augustin, Bischof von Hippo, schreibt 408 an den Donatistenbischof Vincentius: „Du meinst, man dürfe niemanden zur Gerechtigkeit zwingen, obwohl du liest, daß der Hausvater zu seinen Knechten gesagt hat: ,Alle, die ihr findet, zwinget einzutreten', und obwohl du liest, daß auch Saulus, der spätere Paulus, unter dem Zwange einer gewaltsamen

${ }_{12}$ Karl Heinz Chelius, Compelle intrare, in: Augustinus-Lexikon 1 (Basel 1986-1994) 1084f.

13 Deutscher Text nach Joseph Franz von Allioli, Das Neue Testament, deutsch nach der Vulgata (Neuausgabe Colmar 1942) 219.

14 Die Bibel... nach der Übersetzung Martin Luthers, Württembergische Bibelanstalt (Stuttgart 1968) Das Neue Testament, 100.

15 Wie Anm. 13.

${ }^{16}$ Einheitsübersetzung der Heiligen Schrift. Das Neue Testament (Stuttgart $\left.{ }^{6} 1975\right) 151$. 
Einwirkung Christi zur Erkenntnis und Annahme der Wahrheit gebracht worden ist... Du begreifst also bereits, wie ich meine, daß es nicht darauf ankommt, ob jemand überhaupt gezwungen wird, sondern wozu er gezwungen wird, mag es gut oder böse sein.“ Viele Häretiker, so argumentiert Augustinus, hätten durch Zwang den Weg zur Kirche zurückgefunden. „Diesen Beispielen, die mir von meinen Mitbischöfen vorgehalten wurden, habe ich mich gebeugt." Diskret weist Augustinus in diesem Brief darauf hin, daß er früher anders gedacht habe. Er sei der Meinung gewesen, niemand dürfe zur Einheit in Christus gezwungen werden. Aber die praktische Erfahrung mit der nordafrikanischen Donatistenbewegung habe ihn eines Besseren belehrt. Umsichtiger Zwang könne unter Umständen zur Bekehrung der vom gemeinsamen Glauben Abweichenden - und damit zum öffentlichen Frieden - beitragen ${ }^{17}$.

Damit war ein neues Paradigma geschaffen. Fortan wurde das Gleichnis vom großen Gastmahl ausschließlich im geschilderten Sinne interpretiert. Augustins Deutung hat in der folgenden Zeit vor allem beim Umgang mit Glaubens-Abweichungen in der Kirche, bei der Behandlung von Häresien und Häretikern eine breite, oft verhängnisvolle Wirkung entfaltet. Dabei wurde bald auch die Grenze überschritten, die Augustin noch eingehalten hatte und eingehalten wissen wollte - lehnte er doch die Todesstrafe gegen Häretiker auch in seinen späteren Jahren entschieden ab. In den Ketzerprozessen des Mittelalters jedoch - die erste Häretiker-Hinrichtung fand im Westen im Jahr 385 am Trierer Kaiserhof statt - fiel der altchristliche Verzicht auf Körperstrafen gegenüber Häretikern Zug um Zug dahin. Die Gelassenheit früherer Zeiten, die sich am biblischen Gleichnis vom Unkraut unter dem Weizen orientiert und biblisch zur Geduld geraten hatten („Laßt beides wachsen, bis die Ernte kommt“), geriet immer mehr in Vergessenheit. An ihre Stelle trat ein richterlicher Dezisionismus, der im „Gottesfrevel“ des Häretikers und der Verbreitung seiner Lehren ein unmittelbar und augenblicklich zu sühnendes Verbrechen sah.

So konnte Joseph Höffner in seinem Buch „Kolonialismus und Evangelium“ schon 1947 über die neue Auslegung des compelle intrare durch Augustinus das scharfe Urteil fällen: „Diese Sätze wurden von Jahrhundert zu Jahrhundert weitergegeben; sie wurden ins Gratianische Dekret aufgenommen; sie dienten zur Rechtfertigung der Ketzerbestrafung, der Inquisition und der Unterjochung der Indianer noch im 16.Jahrhundert." ${ }^{18}$ Arnold Angenendt hat in seinem kürzlich erschienenen Buch „Toleranz und Gewalt“ die im Mittelalter in der Kirche üblich werdende, in der frühen Neuzeit perfektionierte Praxis der Häretikertötung den „christlichen Sündenfall“ schlechthin genannt ${ }^{19}$.

17 Ep 93: Ad Vincentinum (CSEL 34). Deutsche Übersetzung: Alfred Hoffmann, Des heiligen Kirchenvaters Aurelius Augustinus Ausgewählte Briefe (= Bibliothek der Kirchenväter Bd.IX, Kempten, München 1917) 333-384, die Zitate 338, 349f.

18 Joseph Höffner, Kolonialismus (wie Anm. 8) 45.

19 Arnold Angenendt, Toleranz und Gewalt. Das Christentum zwischen Bibel und Schwert (Münster ${ }^{2} 2007$ ) 245. 


\section{Gewaltabwehr und neue Gewalt: die Kreuzzüge}

Haben auch die Kreuzzüge mit dem compelle intrare zu tun? Gewiß nicht unmittelbar. Denn sie gehörten einem anderen Genus an als das Vorgehen gegen Ketzer, sie waren - zumindest im Verlauf und im Ergebnis - regelrechte Kriege, in denen sich die abendländische Christenheit gegen äußere Feinde wandte ${ }^{20}$. Es waren Kriege gegen Heiden draußen, nicht Kämpfe gegen Häretiker drinnen. Die christlichen Ritter wollten die Muslime, die sich der Heiligen Stätten bemächtigt hatten, nicht bekehren. Dennoch zeigt sich in mehreren Punkten eine verwandte Tendenz.

Daß die Eroberung Palästinas durch den Islam christlichen Pilgern den Weg zu den Heiligen Stätten versperrte, empfanden viele Christen im Abendland als eine schwer erträgliche Demütigung. So begannen die Kreuzzüge „mit dem Doppelziel, die Christen im Osten von der Herrschaft der Moslems zu erlösen und das Heilige Grab in Jerusalem zu befreien "21. In seiner Kreuzzugspredigt in Clermont-Ferrand (1095) - einem höchst wirkungsvollen Aufruf, wenn man an die umwälzenden Folgen denkt! - hob Papst Urban II. auf zwei Dinge ab: den Hilferuf der byzantinischen Christen, die von den Muslimen bedrängt und angegriffen würden, und die Notwendigkeit ihrer Befreiung durch ein Heer von Kreuzfahrern, die durch päpstliche Ermächtigung und geistlichen Lohn zu milites Christi, Soldaten Christi, werden sollten. Ihnen wurde eine Generalabsolution erteilt, wenn sie sich auf den Weg nach Osten machten. Wer in wahrer Buße fiel, der sollte Vergebung seiner Sünden und die Frucht ewigen Lebens erhalten ${ }^{22}$.

Einzelheiten brauchen hier nicht ausgeführt zu werden, da sich bei dieser Tagung ein eigener Vortrag den Kreuzzügen widmen wird. Hier sei nur auf den Gegenwartsaspekt des Themas hingewiesen. Die Zeit der Kreuzzüge hat nicht nur das Verhältnis Christentum - Islam dauerhaft belastet (und ebenso das Verhältnis zwischen Christen und Juden wegen der heimischen Pogrome in Kreuzzugszeiten!). Sie bot dem Islam auch Gelegenheit, eigene Angriffe und Eroberungen als „Verteidigungskriege" zu deklarieren, und das bis zum heutigen Tag. Bis heute werden Christen in der muslimischen Terminologie zugespitzt als „Kreuzzügler“ bezeichnet - und wenn heute George W. Bush von „Kreuzzügen gegen den Terror“ spricht (wie schon Dwight D. Eisenhower vom „Kreuzzug in Europa“ sprach), so hören Muslime in diesem Wort nicht etwa eine abgeblaßte, säkularisierte Formel (wie bei

\footnotetext{
20 Allgemeine Literatur: Steven Runciman, Geschichte der Kreuzzüge (München ${ }^{32001) ; ~ H a n s ~}$ Eberhard Mayer, Geschichte der Kreuzzüge (Stuttgart $\left.{ }^{10} 2005\right)$. - Zur Begrifflichkeit: Ernst Dieter Hebl, Was ist eigentlich ein Kreuzzug?, in: Historische Zeitschrift 259 (1994) 297-336; Kaspar Elm, Die Kreuzzüge, Kriege im Namen Gottes? (= Kirche und Gesellschaft 231, Köln 1996).

21 Victor Conzemius, Die Kreuzzüge, in: Communio 31 (2002) 133-142 (134).

22 Die Rede ist von verschiedenen Chronisten überliefert, von denen einige mit Ausschmückungen und frei erfundenen Passagen arbeiten. Als authentischster Text gilt derjenige, den der Chartreser Priester und Geschichtsschreiber Fulcher, der Kapellan des späteren Königs Balduin von Jerusalem - wahrscheinlich ein Augenzeuge bei der Synode in Clermont -, überliefert hat: Historia Hierosolymitana, ed. H. Hagenmeyer (Heidelberg 1913).
} 
„Kreuzzügen“ gegen Hunger, Kälte, Armut, Drogenmißbrauch), sondern sie erinnern sich an konkrete Ereignisse, die bis heute ihr Geschichtsbild prägen.

Umgekehrt wird heutigen Europäern, wenn sie den Kreuzzugsaufruf Papst Urbans lesen, der Anklang an aktuelle Dschihad-Aufrufe aus islamischen Ländern der Gegenwart nicht entgehen. Vor allem der Hinweis auf den unmittelbar bevorstehenden Paradieseslohn erinnert an Äußerungen aus dem Kreis islamischer, sich selbst opfernder Krieger von heute ${ }^{23}$. Im Vorübergehen sei angemerkt, daß diese Krieger, die wir im Westen als „Selbstmordattentäter“ zu bezeichnen pflegen, inzwischen fast in der ganzen islamischen Welt (auch in Ländern mit laizistischer Verfassung wie der Türkei) als „Martyrer“ bezeichnet werden. Das ist beunruhigend, zeigt es doch, daß hier eine ursprüngliche Gemeinsamkeit im Martyrerverständnis, die Judentum, Christentum und Islam lange Zeit verband, - die Überzeugung nämlich, daß man das Martyrium nicht selbst suchen dürfe, daß Martyrium und Selbstmord einander ausschlössen ${ }^{24}$ - offenkundig in unserer Zeit zu Ende geht.

\section{Kolonialismus und Christentum}

Die Kreuzzüge waren nicht nur ein Ausgriff der europäischen Christenheit nach draußen, der zu schärferen Abgrenzungen gegenüber der nichtchristlichen Welt führte. Sie standen zugleich in Verbindung mit Vorgängen im Inneren Europas. Die christlich geformte europäische Welt konsolidierte sich vom 11. bis zum 13. Jahrhundert, entwickelte spezifische Gemeinsamkeiten und bereitete die Grundlagen für Europas spätere weltweite Machtstellung vor. Ein Dualismus entwickelte sich: Gottesfriede im Inneren als Vorstufe der späteren Landfrieden und des sich entfaltenden staatlichen Rechts- und Friedensraumes mit seiner Zähmung und Kultivierung der Gewalt ${ }^{25}$ - Krieg (oder gewaltsame Bekehrung) im Äußeren, gegenüber den Heiden, der nichtchristlichen Welt ${ }^{26}$. Im Zeitalter kolonialer Expansion wurde die christliche Ethik immer mehr zu einer europäischen Binnenethik. Sie galt nicht mehr schlechthin und überall; „beyond the line“ hatte sie meist ihr Recht verloren. Vielfältig wurde die im Inneren Europas „gezähmte“ Gewalt nach draußen gekehrt, ihre Antriebsrichtung veränderte sich. Jenseits des Äquators

\footnotetext{
23 Mark Juergensmeyer, Terror in the Mind of God. The Global Rise of Religious Violence (London 2000) 165ff., $218 \mathrm{ff}$; Joseph Croitorou, Der Märtyrer als Waffe. Die historischen Wurzeln des Selbstmordattentats (München 2003) 71ff., $121 \mathrm{ff} ., 188 \mathrm{ff} . ;$ Diego Gambetta (Hrsg.), Making Sense of Suicide Missions (Oxford 2005) $131 \mathrm{ff} ., 233 \mathrm{ff} ., 259 \mathrm{ff}$.

24 Hierzu Hans Maier, Politische Martyrer? Erweiterungen des Martyrerbegriffs in der Gegenwart, in: Stimmen der Zeit 222 (2004) 291-305.

25 Wolfgang Reinhard, Geschichte der Staatsgewalt (München ${ }^{3} 2002$ ).

${ }^{26}$ Eberhard Schmitt, Dokumente zur Geschichte der europäischen Expansion I-VI (V und VI gemeinsam mit Thomas Beck) (München 1986-1988; Wiesbaden 2003-2008); Thomas Beck, Marília dos Santos Lopes, Christian Rödel (Hrsg.), Barrieren und Zugänge. Die Geschichte der europäischen Expansion. FS für Eberhard Schmitt zu seinem 65. Geburtstag (Wiesbaden 2004).
} 
wurde der Europäer oft genug - nach der sprichwörtlichen Formulierung Guillaume de Raynals - zum „gezähmten Tiger, der in den Wald zurückkehrt“.

Gewiß, die Kreuzzüge brechen im späteren Mittelalter ab und verschwinden schließlich ganz. Sie passen nicht mehr in die Zeit, sind ein Stück Vergangenheit, werden zu Gegenständen ritterlicher Nostalgie ${ }^{27}$. Dennoch hören nicht alle Kontinuitäten auf. Zu den Antrieben der kolonialen Expansion seit dem 14.Jahrhundert gehört gewiß immer noch das christliche „Geht hinaus in alle Welt“, die Sorge um die Bekehrung der Völker - nunmehr freilich vermischt mit anderen Elementen: einer generellen Neugier bei der Erkundung der Welt, der Erforschung der Seewege in andere Kontinente, der Suche nach dem mystischen Dorado, dem Goldland, nach Gewürzen, Sklaven, Abenteuern ${ }^{28}$. Im Kolonialzeitalter folgt dem christlichen Missionar fast unvermeidlich der Soldat, der Eroberer, der Kaufmann, der Verwalter auf dem Fuß. Immer unablösbarer verbindet sich die christliche Mission mit anderen, weltlichen Zwecken. Sie wird europazentrisch und bleibt es lange. In der neuzeitlichen vatikanischen Verwaltung wird die Congregatio de propaganda Fide lange, bis ins 20.Jahrhundert hinein, fast ausschließlich von Europäern beherrscht ${ }^{29}$.

Der Schwerpunkt der theologischen Debatte verlagert sich in dieser Zeit in das öffentliche Recht, das ius gentium - in die Frage des Umgangs mit den Eingeborenen der Neuen Welt, ihren Rechten und Pflichten. Joseph Höffner hat gezeigt, daß die Härte der Kriege gegen die Eingeborenen in der Neuen Welt auch mit der Verschärfung der Lehre vom „gerechten Krieg“ zusammenhing, die sich schon in der Zeit der Kreuzzüge vorbereitet hatte. So lehrte bereits Papst Innozenz IV. (1243-1254), der Papst könne den Ungläubigen befehlen, christliche Glaubensboten in den Ländern ihrer Herrschaft zuzulassen - sollten sie den Gehorsam ver-

${ }^{27}$ Nach wie vor die subtilste Darstellung der politischen und militärischen Bedeutung des Rittergedankens im ausgehenden Mittelalter und der Wandlungen des Kreuzzugsideals: Jan Huizinga, Herbst des Mittelalters (Kröner Taschenausgabe Band 204, Stuttgart 1969) 126-146.

28 Eberhard Schmitt, Die Anfänge der europäischen Expansion (Idstein 1991); Urs Bitterli, Eberhard Schmitt (Hrsg.), Die Kenntnis beider „Indien“ im frühneuzeitlichen Europa (München 1991); Thomas Beck, Annerose Menninger, Thomas Schleich (Hrsg.), Kolumbus' Erben. Europäische Expansion und überseeische Ethnien im ersten Kolonialzeitalter 1415-1815 (Darmstadt 1992); Horst Gründer, Welteroberung und Christentum. Ein Handbuch zur Geschichte der Neuzeit (Gütersloh 1992); Eberhard Schmitt, Atlantische Expansion und maritime Indienfahrt im 16.Jahrhundert (Bamberg 1992); ders., Friedrich-Karl von Hutten, Das Gold der Neuen Welt. Die Papiere des Welser-Konquistadors und Generalkapitäns von Venezuela Philipp von Hutten 1534-1541 (Hildburghausen 1996); Klaus Koschorke (Hrsg.), „Christen und Gewürze“. Konfrontation und Interaktion kolonialer und indigener Christentumsvarianten (Göttingen 1998); Jürgen Osterhammel, Sklaverei und die Zivilisation des Westens (= Carl Friedrich von Siemens Stiftung, Themen Band 70, München 2000); Markus A. Denzel (Hrsg.), Vom Welthandel des 18. Jahrhunderts zur Globalisierung des 21. Jahrhunderts (Stuttgart 2007).

${ }^{29}$ Die Congregatio de Propaganda Fide wurde 1622 von Gregor XV. als Zentrale für die katholische Missionstätigkeit geschaffen und umfaßte im Jahr 1962577 Diözesen, 6 Abteien nullius, 114 Apostolische Vikariate, 93 Apostolische Präfekturen und 3 Missionen sui iuris; vgl. Nikolaus Kowalsky, Propaganda-Kongregation, in: Lexikon für Theologie und Kirche, Band 8 (Freiburg $\left.{ }^{2} 1963\right)$. 
weigern, seien sie mit weltlicher Gewalt zu zwingen. Möglicher Grund für einen gerechten Krieg war also nicht mehr nur das von Heiden gegen Christen begangene Unrecht, es war vielmehr das Unrecht (des Nichtglaubens) gegen Gott schlechthin. Theoretisch war damit „das Verhältnis zu den Heidenvölkern als dauernder Kriegszustand gekennzeichnet " 30 .

Gegen diese Verengung und Zuspitzung des biblischen „Nötige sie!“ erhob sich immer wieder Protest auch unter christlichen Theologen und Juristen. Gerade das Spanien der Kolonialzeit ist davon erfüllt - man denke an den Kampf des Las Casas gegen die Übergriffe der Konquistadoren und an die Entwicklung einer neuen Kolonialethik durch Vitoria. Doch es brauchte noch lange, bis sich die Kirche von dem inzwischen immer mehr profan gewordenen, nicht mehr religiös unterfangenen Kolonialismus zu lösen begann.

Im Grund hat erst das späte 19. und das 20. Jahrhundert diesen Punkt erreicht. In dieser Zeit begannen die Päpste, beginnend mit Leo XIII., Kolonialismus und Mission zu entkoppeln, die Mission, so gut es ging, von weltlichen Einflüssen zu befreien und mit Energie einen einheimischen Klerus aufzubauen ${ }^{31}$. In der Folgezeit wuchs die Kirche konsequent in weltweite, nicht mehr auf Europa zentrierte Dimensionen hinein. Die Zusammensetzung des Kardinalskollegiums - bis 1961 noch mit italienischer Mehrheit - änderte sich grundlegend, ebenso veränderten sich die Episkopate der früher sogenannten Missionsländer. Hanno Helbling hat den Unterschied 1981 so beschrieben: „Vor dreißig Jahren betrat, wenn er Rom besuchte, der Missionsbischof europäischer oder amerikanischer Herkunft mit farbigem Gefolge den Palazzo der Kongregation De Propaganda Fide an der Piazza di Spagna. Heute schreitet der ,eingeborene ordinarius loci dort seinem weißen Sekretär und Berater voran. "32 Helbling spricht von einem „Abschied vom alten Missionsdenken“. Er ist eine Konsequenz aus der Tatsache, daß heute die „Dritte Welt“ in der Katholischen Kirche den Rang der früheren „Ersten Welt“ erreicht hat. Dementsprechend heißt die Missionskongregation seit 1988 auch nicht mehr „de Propaganda Fide“, sondern „pro Gentium Evangelizatione“. Der paternalistische europäische Akzent im Namen der Missionszentrale gehört der Vergangenheit an.

\section{Compelle intrare - auch in der Reformation?}

Mehr im Vorübergehen, in Form eines Exkurses, sei an dieser Stelle nach dem Beitrag der Reformation zu unserem Thema gefragt. Wie haben die Reformatoren das

30 Höffner, Kolonialismus (wie Anm. 8) 69.

31 Diese Absichten waren schon in dem von Francesco Ingoli, dem ersten Sekretär der Propaganda-Kongregation (1622-1649), entworfenen Programm enthalten, konnten systematisch jedoch erst realisiert werden, nachdem das Ende des Kirchenstaates 1871 die Kirche von der unmittelbaren Rücksicht auf die traditionellen europäischen Schutzmächte entbunden hatte.

32 Hanno Helbling, Politik der Päpste. Der Vatikan im Weltgeschehen 1958-1978 (Frankfurt 1981) 63. 
Verhältnis von Wahrheit und Freiheit, das Problem des Schutzes und der Ausbreitung des Glaubens gesehen? Gibt es auch ein reformatorisches compelle intrare?

Man muß die Frage differenziert beantworten ${ }^{33}$. So hat Luther die Freiheit des Glaubensaktes betont, zugleich aber am absoluten Anspruch des Evangeliums festgehalten. „Toleranz“ - diese Eindeutschung des lateinischen Wortes tolerantia geht auf ihn zurück - war für ihn Sache der Liebe, des duldenden Einverständnisses, nicht aber Sache des Glaubens, der „intolerant“ blieb und bleiben mußte. Auch bei Calvin gehört tolerantia dem Bereich des praktischen Zusammenlebens an, sie ist mansuetudo animi, ein Weg der Mitte zwischen übermäßiger Strenge und alles verzeihender Milde. Duldung kann es aber nicht geben gegenüber Götzendienst, Aberglauben und Magie, auch nicht gegenüber Abweichungen von zentralen Lehren des christlichen Glaubens wie dem trinitarischen Dogma; in diesem Sinn hat Calvin, wie bekannt, die Hinrichtung des spanischen Arztes Servet im Jahr 1553 in Genf gerechtfertigt.

Steht so der Altprotestantismus, von wenigen Ausnahmen abgesehen, noch ganz auf dem Boden des einheitlich-allgemeinen Glaubens und seiner das öffentliche Leben verpflichtenden Geltung ${ }^{34}$, so rückte die staatliche Praxis unter dem Druck der Umstände bald von dieser Haltung ab. Bei den Einsichtigen wuchs die Erkenntnis, daß es sich beim Streit zwischen Katholiken und Protestanten nicht um den traditionellen Glaubensstreit zwischen einer Mehrheit und einer häretischen Minderheit handelte, mit dem die Theologie seit Augustinus (ja schon seit Paulus) immer wieder konfrontiert war. Es sind vielmehr vergleichbar starke ReligionsCorpora, die einander gegenüberstehen - und beide berufen sich auf das christliche Erbe. Protestanten und Katholiken können einander, das zeigt sich bald, weder verdrängen noch vernichten noch bekehren, so daß sie notwendigerweise Frieden miteinander halten müssen, Religionsfrieden - zumindest bis ein Allgemeines Konzil das Problem „spaltiger Religion“ gelöst hat ${ }^{35}$.

Der Augsburger Religionsfrieden war ein entscheidender Schritt zur faktischen Duldung der katholischen und der lutherischen Konfession im Reich. Er bildete noch keinen Auftakt zu einer allgemeinen Religionsfreiheit; sein Ziel war, nach der

33 Zum Folgenden: Klaus Schreiner, Gerhard Besier, Toleranz (wie Anm. 10); bes. die Abschnitte VIII-X (472-494) „Toleranz und Intoleranz im Zeitalter des Humanismus, der Reformation und Gegenreformation“, „,Toleranz“ als Handlungsbegriff im 16.Jahrhundert“ und „Politische Theorie, Reichs- und Kirchenrecht um 1600“ (Klaus Schreiner) sowie XI (495-510) „,Toleranz“ als religionspolitischer Begriff im 17. und 18. Jahrhundert“ (Gerhard Besier).

34 Ernst Walter Zeeden, Die Entstehung der Konfessionen (München 1965) 11, bemerkt für die Anfänge der Konfessionsbildung zurecht: „... jede Konfession war ihrer Intention nach universal: Jede verstand sich als Confessio Catholica und bezeichnete sich in ihren authentischen Verlautbarungen so."

35 Dietmar Willoweit, Religionsrecht im Heiligen Römischen Reich zwischen Mittelalter und Aufklärung, in: Als Frieden möglich war. 450 Jahre Augsburger Religionsfrieden, hrsg. von Carl A. Hoffmann u.a. (Regensburg 2005) 35-50; Martin Heckel, Vom Religionskonflikt zur Ausgleichsordnung. Der Sonderweg des deutschen Staatskirchenrechts vom Augsburger Religionsfrieden 1555 bis zur Gegenwart (= Bayerische Akademie der Wissenschaften, phil.-hist. Kl., Abhandlungen NF Heft 130, München 2007). 
griffigen Formel von Gerhard Anschütz, „nicht Glaubensfreiheit, sondern Glaubenszweiheit“. Trotz der im Geist der Zeit ständisch (korporativ) konzipierten Form der religiösen Freiheit enthielt der Augsburger Religionsfriede doch Bestimmungen, die der persönlichen Entscheidung etwas Raum ließen, wie etwa das Recht, mit Weib und Kind gegen Bezahlung einer Nachsteuer auszuwandern $(\mathbb{2} 24)^{36}$. Erheblich weiter zieht dann ein Jahrhundert später, nach der Erschöpfung der konfessionellen Leidenschaften im Dreißigjährigen Krieg, das Instrumentum Pacis Osnabrugense den Kreis der freien Religionsübung. In Art. V $\ 31$ und 32 IPO wird bestimmt, daß die andersgläubigen Stände, die im „Normaljahr“ 1624 das exercitium religionis nach katholischer oder Augsburger Konfession besessen haben, dies auch in Zukunft behalten sollten; die es nicht besitzen oder künftig zur anderen Religion übertreten wollen, sollen vom Landesherrn „in Geduld ertragen werden" (patienter tolerentur) und in Gewissensfreiheit - hier taucht der Begriff der conscientia libera im Reichsrecht auf! - ihre Hausandacht pflegen und im benachbarten Gebiet dem öffentlichen Gottesdienst beiwohnen können ${ }^{37}$.

Daß vor diesem Hintergrund das compelle intrare im evangelischen Bereich allmählich verblassen mußte, dafür mag hier ein spätes Zeugnis aus der ersten Hälfte des 18. Jahrhunderts stehen. Unter dem Präsidium des Theologen und Kanzlers Christoph Matthäus Pfaff verteidigte Wolfgang Ludwig Liesching, ein aus Göppingen stammender Tübinger Stiftler, am 21. März 1732 in einer Disputatio in der Tübinger Universität Thesen „De tolerandis vel non tolerandis in Religione Dissentientibus“38. Ausgangspunkt war wiederum das lukanische „Große Gastmahl“ und die zentrale Weisung anankason eiselthein - compelle ad intrandum.

Der Text ${ }^{39}$ wird ganz im Sinn der Tradition ausgelegt: Der Paterfamilias ist Gott selbst; der Knecht, der die Geladenen ruft, ist Christus mit den Aposteln, das Haus ist die Kirche, das große Gastmahl die Gnade, die Einladung die Predigt des Evangeliums. Die Erstgeladenen, die die Einladung verschmähen, sind die Juden, die Nachfolgenden sind die Christen aus allen Völkern, die dem Ruf des Knechtes folgen. Das alles ist, wie der Kommentator sagt, leicht zu erkennen und mit anderen Parallelen im Neuen Testament zu vergleichen ${ }^{40}$.

Die zentrale These der Schrift wird gleich zu Anfang entwickelt: Die Worte anankazein und compellere, so der Verfasser, seien strikt im Sinn einer „emphatischen und drängenden Einladung“, einer invitatio emphatice $\mathcal{E}$ instantissime facienda, auszulegen - keinesfalls jedoch seien Zwang und Gewalt gemeint. Auch sei die Gleichsetzung des „Knechtes“ mit der weltlichen Gewalt eine Mißdeutung

\footnotetext{
36 Vgl. Axel Gotthard, Der Augsburger Religionsfrieden (Münster 2004) 118ff., der betont, daß das Ius emigrandi 1555 zwischen den Konfessionen nicht strittig war.

37 Willoweit, Religionsrecht (wie Anm.35) 47ff.; Heckel, Vom Religionskonflikt (wie Anm.35) $25 \mathrm{ff}$.

38 Im folgenden zitiert nach UB München Sig. $4^{\circ}$ Theol. 2896. Für den Hinweis auf diese Schrift danke ich Klaus Schreiner.

3939 Commentariolus Theologicus ad Verba Christi Compelle ad intrandum sive De tolerandis vel noch tolerandis in Religione Dissentientibus... (Tubingae 1732).

40 Commentariolus $\$ 1$; genannt wird u. a. die Parallelstelle Math 22, $1 \mathrm{ff}$.
} 
des Gleichnisses, gemeint seien Christus und die Apostel, diese aber hätten mit ihrer Predigt niemals äußere Gewalt oder gar Waffen (vim externam vel carnalia arma) verbunden. Es versteht sich, daß von diesem Ausgangspunkt her die ganze Geschichte des compelle intrare kritisch gesehen und als Verirrung gebrandmarkt wird, die längst durch bessere Erfahrungen mit Toleranzregeln widerlegt sei ${ }^{41}$. Daß auch in der Frühzeit der Reformation Gewalt gegen Häretiker geübt wurde, wird zum Einzelfall erklärt, den man nicht der protestantischen Gemeinschaft im ganzen vorwerfen könne ${ }^{42}$.

\section{Ein Schlußpunkt: der Kampf um die Religionsfreiheit im Zweiten Vatikanischen Konzil}

Länger als im Protestantismus hat sich das compelle intrare in der katholischen Welt gehalten. Freilich war Gewalt zum Schutz der Religion seit dem 19.Jahrhundert nur noch in katholischen Staaten durchsetzbar - und auch hier keineswegs überall und unbeschränkt. Als Pius IX. 1864 in seinem "Syllabus" mit anderen „Zeitirrtümern“ auch die Religionsfreiheit verurteilte ${ }^{43}$, versuchte der französische Bischof Dupanloup den Inhalt dieser Schrift durch eine „weitherzige“ Interpretation zu entschärfen. Er unterschied die These von der Hypothese: Als allgemeiner Grundsatz (These) sei die Religionsfreiheit nicht annehmbar; als Hypothese dagegen, unter besonderen geschichtlichen Bedingungen, zum Beispiel in einem religiös gespaltenen Land, könne sie durchaus berechtigt sein. Die damit skizzierte Verteidigungslinie - sie fand übrigens den Beifall des Papstes! - war freilich problematisch und trug nicht weit. Sie öffnete dem Opportunismus und der Mehrdeutigkeit Tür und Tor. Der Pariser Witz hat sich der Lage rasch bemächtigt; anspielend auf das ziemlich weltliche Leben des Nuntius Chigi, sagte man: „Die These ist, wenn der Nuntius erklärt, man müsse die Juden verbrennen; die Hypothese, wenn er bei Herrn Rothschild zu Mittag ißt." “44

Ein letztes und entscheidendes Mal wurde in jüngerer Zeit in der katholischen Welt über Gewalt zum Schutz oder zur Ausbreitung des Glaubens theologisch gerungen, nämlich während des Zweiten Vatikanischen Konzils, bei der Erklärung

${ }^{41}$ Commentariolus $\$ \mathbb{S} 4$, 5. - Gegenüber offenkundigen Atheisten, Spinozisten, Deisten gilt es freilich auch nach Meinung des Autors die „Grenzen der Toleranz zu ziehen“ (limites tolerantiae ponere), so $₫ 6$, Anm. (u) - ohne daß genauer gesagt wird, was mit ihnen zu geschehen habe.

${ }_{42}$ Corollarium I; hier werden die durch Feuer und Schwert hingerichteten Ketzer Servet, Valentinus Gentilis und Johannes Sylvanus erwähnt mit dem entschuldigenden Zusatz: facta haec particularia sunt, quae Protestantium toti Ecclesiae nequeunt objici.

43 Text: ASS 3 (1867) 168-176; vgl. Hans Maier, Die Freiheitsidee der Aufklärung und die katholische Tradition, in: Krzysztof Michalski (Hrsg.), Aufklärung heute. Castelgandolfo-Gespräche 1996 (Stuttgart 1996) 75-106; Hubert Wolf, Der Syllabus, in: Kirche im 19.Jahrhundert, hrsg. von Manfred Weitlauff (Regensburg 1998) 115-139.

${ }^{44}$ Félix-Antoine-Philibert Dupanloup, La convention du 15 septembre et l'encyclique du 8 décembre (Paris 1865); Roger Aubert, Le Pontificat de Pie IX (Paris 1952) 252. 
über die Religionsfreiheit. Die Debatte in der 80.Generalkongregation im September 1964 wurde zu einer Zerreißprobe; über keinen Text des Zweiten Vatikanums, nicht einmal über die gleichfalls umkämpfte Pastoralkonstitution über die Kirche in der Welt von heute (Gaudium et Spes), ist in der Konzilsaula erbitterter räsoniert und gestritten worden ${ }^{45}$.

Eine Mehrheit der Väter stand zwar von Anfang hinter der Erklärung. Aber die opponierende Minderheit war stark, weil sie sich auf unbestreitbar gegensätzliche, dem Textentwurf diametral widersprechende Aussagen des päpstlichen Lehramts stützen konnte - und dies in einer ununterbrochenen Folge von Zeugnissen von der Zeit der Französischen Revolution bis zum Pontifikat Leos XIII. Man monierte, daß in der Erklärung die Rechte des irrenden Gewissens denen des „rechten Gewissens“ gleichgestellt würden. Der Irrtum habe aber gegenüber dem objektiven Anspruch der Wahrheit kein Recht. „Das Schema sündigt im Übermaß, weil es behauptet, daß derjenige der Achtung würdig ist, der seinem Gewissen folgt, auch wenn er sich täuscht“ - so Kardinal Ottaviani. Und Bischof Marcel Lefèbvre verurteilte den Text im ganzen, „weil er nicht auf die Rechte Christi und der Kirche gegründet" sei ${ }^{46}$.

Doch auch die Gegenseite kam zu Wort. Sie führte ins Feld, daß die Praxis des Heiligen Stuhls hinsichtlich der Konkordate sich längst den Prinzipien der Religionsfreiheit angenähert habe. Kardinal Heenan, der Erzbischof von Westminster, wies auf die Verhältnisse in England hin und zeichnete das Bild einer Staatskirche - der anglikanischen -, die ungeachtet ihrer offiziellen Stellung Andersgläubigen Toleranz gewähre. Seine Worte wurden berühmt, weil sie die Situation einer pluralistischen Gesellschaft präzise umschrieben: „Heutzutage kann Großbritannien keinesfalls als katholisch angesehen werden. Die Kirche von England ist die offizielle Staatskirche, an deren Spitze die Königin steht. Tatsächlich aber praktizieren viele unserer Mitbürger keine Religion. Dennoch bezeichnet sich die Mehrzahl der Engländer als Christen. In England sind die Kinder im allgemeinen getauft, die Leute wollen sich meist kirchlich trauen lassen, und fast alle erhalten ein christliches Begräbnis. Natürlich gibt es daneben auch Menschen, die keine Religion bekennen. Wir haben also eine pluralistische Gesellschaft, in der trotz allem die Religion privat und öffentlich geehrt wird. Obwohl die Kirche von England die Staatskirche ist, wird den Bürgern der anderen Bekenntnisse völlige Religionsfreiheit garantiert. Daher gibt der Staat zum Beispiel den katholischen Schulen eine wesentliche Beihilfe und bezahlt vollständig die Gehälter ihrer Professoren, auch wenn es Priester oder Ordensleute sind. Aber - und das ist richtig - die katholi-

${ }^{45}$ Hamer, Congar, Konzilserklärung passim; vgl. besonders die dort enthaltenen Kommentare von Pietro Pavan, Jan Willebrands, Emile-Joseph De Smedt, Jérôme Hamer, John Courtney Murray, Yves Congar und Pierre Benoit; Roman A. Siebenrock, Theologischer Kommentar zur Erklärung über die religiöse Freiheit (Dignitatis humanae), in: Herders Theologischer Kommentar zum Zweiten Vatikanischen Konzil, hrsg. von Wilhelm Hünermann, Bernd Jochen Hilberath, Band 4 (Freiburg 2005) 125-218.

46 Hamer, Congar, Konzilserklärung 86f. 
schen Schulen erfreuen sich der gleichen Rechte und Pflichten wie die Schulen der Kirche von England." ${ }^{4}$

Die Waage schwankte heftig zwischen Befürwortern und Gegnern der Religionsfreiheits-Erklärung. Das Schema wurde mehrmals umgearbeitet. Viele Einzelfragen wurden neu erörtert. Begriffe wie Toleranz, Zwang, Gewissen, Grenzen der Religionsfreiheit wurden präzisiert. Im Oktober 1964 stand die dritte Textfassung fest. Im November sollte die Abstimmung erfolgen. Doch die Gegner des Dokuments erreichten eine Verschiebung. Das löste bei der Mehrheit große Verärgerung aus, die sich in zahlreichen Bittschriften an Papst Paul VI. Luft machte. Dem Text kam die Zwangspause jedoch zugute. Die endgültige Textfassung - es war die sechste - wurde nach weiteren Umarbeitungen und einer wesentlichen Kürzung, Vereinfachung und Konzentration mehr als ein Jahr später, am 7. Dezember 1965, vom Konzil endgültig verabschiedet, nunmehr mit großer Mehrheit: 2308 placet, 70 non placet ${ }^{48}$.

Von da an war die Religionsfreiheit, nachdem sie zum offiziellen Titel eines lehramtlichen Dokuments geworden war, in der katholischen Kirche nicht mehr umstritten, sie war endgültig anerkannt. Es war, wie ein Konzilsberater - Joseph Ratzinger - 1965 formulierte, „das Ende des Mittelalters, ja das Ende der konstantinischen Ära “49.

Positiv war ohne Zweifel, daß das Zweite Vatikanische Konzil das Thema aufgriff und trotz erheblicher Widerstände zu Ende führte; positiv war, daß die alte Zweideutigkeit und Doppelgleisigkeit - alle Rechte für die Wahrheit, für den Irrtum höchstens Duldung - überwunden wurde; positiv war vor allem das Bekenntnis zu Freiheit und Würde des Menschen und die nüchterne Einschätzung des Staates, der in der neuen Sicht der Konzilsväter die Last der Heilssorge verlor, zugleich aber auf seine eigentliche Bestimmung, die Rechts- und Friedewahrung und die Sorge für das öffentliche Wohl, hingelenkt wurde.

Ein Bedauern wird der Historiker freilich äußern: Über den Turbulenzen der Beratungen und Abstimmungen ${ }^{50}$ - und wohl auch aus grundsätzlichen Bedenken - kam die anfangs als einleitender Teil geplante Quaestio historica der Erklärung nicht zustande ${ }^{51}$. Sie hätte die wechselvolle Geschichte des compelle intrare, des staatlichen Patronats über die Kirche, der Unterordnung der „Freiheit“ unter die „Wahrheit“" der halbherzig bejahten und oft wieder zurückgenommenen Toleranz darstellen und erklären sollen; dies wäre freilich nur möglich gewesen, wenn man im Text einen Bruch, eine Abkehr von früheren Gewohnheiten, einen offenen

47 Hamer, Congar, Konzilserklärung 87.

48 Hamer, Congar, Konzilserklärung 115.

49 Joseph Ratzinger, Ergebnisse und Probleme der dritten Konzilsperiode (Köln 1965) 31; zit. bei Konrad Hilpert, Die Anerkennung der Religionsfreiheit, in: Stimmen der Zeit 130 (2005) 809-819 (817).

50 Die insgesamt 32 Abstimmungen über das Schema über die Religionsfreiheit sind dokumentiert bei Hamer, Congar, Konzilserklärung 118-120.

51 Sie wurde in der vierten konziliaren Textfassung beseitigt; Hamer, Congar, Konzilserklärung 102. 
Paradigmenwechsel eingeräumt hätte. Dazu war die Zeit jedoch offenbar noch nicht reif. Die Konzilsväter waren bemüht, Widersprüche zu älteren kirchlichen Erklärungen und Standpunkten zwar nicht zu leugnen oder zu verschleiern, aber sie stellten sie doch nicht offen zur Diskussion. Alles war auf den Eindruck der Kontinuität, der Widerspruchsfreiheit angelegt. Ein historisches Schuldbekenntnis, wie es Papst Johannes Paul II. 35 Jahre später am ersten Fastensonntag des Jahres 2000 ablegen sollte, lag 1965 noch gänzlich außerhalb der allgemeinen Vorstellungen.

Immerhin: Daß die Kirche irren kann und dies auch sagt, ist inzwischen kein Tabu mehr. Und so dürfen wir hoffen, daß die ausstehende Quaestio historica eines Tages von kirchlicher Seite noch nachgeliefert wird! 
